\title{
Quantitative Analysis of Correlated 3D Strontium Titanate Datasets Collected by TriBeam and Diffraction Contrast Tomography
}

\author{
W.C. Lenthe ${ }^{1}$, M.P. Echlin ${ }^{1}$, M. Syha ${ }^{2,3}$, A. Trenkle ${ }^{2}$, P. Gumbsch ${ }^{2,4}$, T.M. Pollock ${ }^{1}$ \\ 1. Materials Department, University California at Santa Barbara, Santa Barbara, USA \\ 2. Institute for Applied Materials IAM, Karlsruhe Institute of Technology, Karlsruhe, Germany \\ 3. European Synchrotron Radiation Facility, Grenoble, France. \\ 4. Fraunhofer IWM, Freiburg, Germany
}

Much attention has been paid to the idea of the correlation of analytical techniques such as tomography. Recently, techniques for the acquisition of 3D tomographic and 4D time resolved datasets have emerged allowing for the analysis of $\mathrm{mm}^{3}$ volumes of material with $\mathrm{nm}$-scale resolution. The TriBeam technique permits the acquisition of 3D EBSD datasets using a femtosecond laser to section material at unprecedented speed [1-2] with low damage [3-4] and high resolution [5]. Diffraction contrast tomography (DCT) [6], a synchrotron based X-ray technique acquires datasets non-destructively, permitting the repeated imaging of samples to collect 4D microstructural evolution [7] in crystalline materials. However, the vast majority of materials tomography datasets have been combined in a purely qualitative sense to date. In this work, a methodology for the precise alignment of tomographic datasets, including the alignment of the sample and orientation reference frames, and simultaneous identification and linking of grain structure between tomography datasets has been developed. The application of these algorithms to a pair of datasets collected from a single strontium titanate (STO) sample using both TriBeam tomography and synchrotron X-ray diffraction contrast tomography (DCT) will be presented. The resulting merged datasets have been quantitatively analyzed on the voxel scale and at the grain scale for the direct comparison of these two tomography techniques.

Tomograms from a single sample were collected by DCT with time evolution and subsequently using the TriBeam, then merged onto one sampling grid for simultaneous quantitative analysis at both the grain and voxel scale. The two tomographic techniques employed collect diffraction information in fundamentally different ways. DCT illuminates the entire sample and simultaneously collects projections on a detector from all grains with orientations satisfying the Bragg condition. In contrast, EBSD patterns are collected serially for each voxel in the TriBeam experiment. Therefore, orientation reference frames and sample reference frames must be aligned, as shown in Figure 1, before quantitative analysis may be performed.

Analysis of the merged datasets shows the resolution limits of the DCT technique and locations where the accuracy of the technique may be degraded. Errors in EBSD segmentation are also identified. Visualizations showing the difference in spatial locations of grain boundaries and centroids will be presented as well as quantitative comparisons of metrics for the quality of the dataset alignment algorithms.

References:

[1] M. P. Echlin et al, Review of Scientific Instruments 83(2) (2012), p. 023701.

[2] M.P. Echlin et al, Advanced Materials 23 (2011), p. 2339.

[3] S. Ma et al, Met. Mat. Trans. A 38 (2007), p. 2349. 
[4] Q. Feng et al, Scripta Mat. 53 (2005), p. 511.

[5] M.P. Echlin et al, Materials Characterization: Tutorial Review 100 (2015), p. 1.

[6] H.F. Poulsen et al, Journal of Applied Crystallography 34 (2001), p. 751.

[7] D. Gonzalez et al, Acta Materialia 61 (2013), p. 7521.

(a.) DCT Experiment

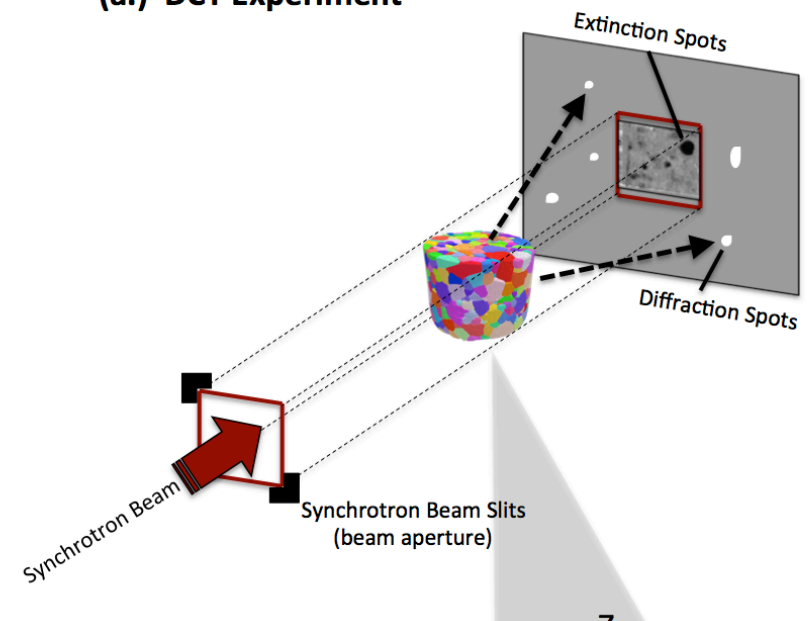

(b.) TriBeam Experiment

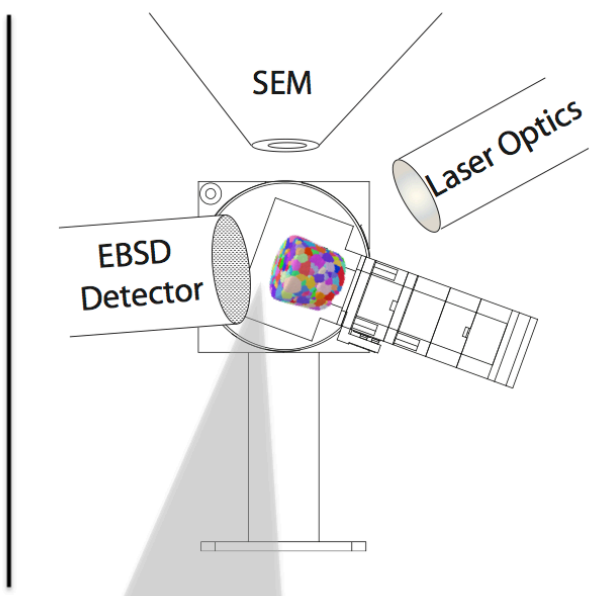

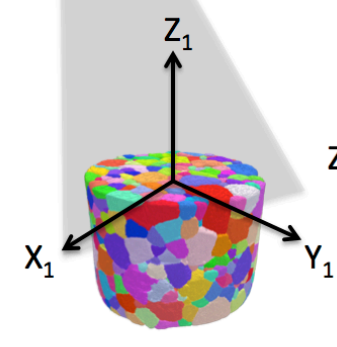

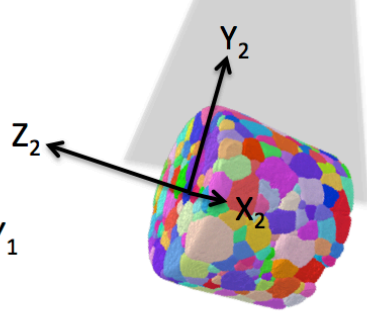

Figure 1: The reference frames are not aligned between the TriBeam tomogram (a) and the DCT tomogram (b), which must be aligned before quantitative analysis of the correlated microstructure can be performed. 